\title{
Long-term follow-up in newborn screening: the role of collaboration
}

\author{
Melissa P. Wasserstein, $\mathrm{MD}^{1}$
}

Newborn screening (NBS) began more than 50 years ago after Robert Guthrie's discovery that phenylketonuria, a preventable form of mental retardation, could be accurately and inexpensively detected in dried blood spotted onto filter paper. ${ }^{1}$ Over the ensuing years, there was a gradual increase in the number of disorders on NBS panels through the adaptation of more sophisticated screening methods. We now find ourselves in interesting times, when the technical ability to screen and a strong political presence of advocacy groups are guiding policy about inclusion of disorders on NBS panels. We are also glimpsing the future use of genomic technologies as screening tools. This exciting expansion process is occurring as NBS enters its sixth decade, a period in life often considered to be a time for mature introspection and self-evaluation. Accordingly, it is time to critically appraise the process and assess whether its goals are being met.

What are the goals of NBS? Generally speaking, the goals are simple: to identify affected individuals as early as possible and to improve their outcomes through early treatment. The former goal focuses on the actual testing procedure, whereas the latter focuses on treatment, disease course, and clinical outcome. Thus, NBS is, in reality, an extensive, multifaceted, long-term process in which the actual testing plays a relatively minor role. The first five decades of NBS focused mainly on expanding testing. Developing and enhancing laboratory operations have led to the ability to detect more disorders. The Secretary's Advisory Committee on Heritable Disorders in Newborns and Children and the development of the Recommended Uniform Screening Panel help guide thoughtful decisions about testing expansion. Largely as a result of these efforts, most states have more than quadrupled their NBS panels over the past decade. However, only minimal attention has been paid to the long-term followup and outcomes of those identified. The need for collecting long-term data on outcomes is clear: many new NBS additions have scant scientific evidence to support the risks and longterm benefits of screening for that particular disease. Most practices related to NBS conditions-from the initial decision to include a disorder on an NBS panel to determination of best treatment practices-have been based on empiric observations, anecdotal evidence, and individual experience. This presents a unique conundrum in which public policy mandates screening and follow-up despite a paucity of objective data to guide clinical decision making. Thus, to determine whether we are, in fact, achieving our goal of improving outcomes, we must focus energy on critically assessing our practices to see how screenpositive children are faring through thoughtful and detailed collection of long-term clinical data. As the complexity of NBS disorders increases (e.g., Krabbe disease, X-linked adrenoleukodystrophy), this is more crucial than ever.

Complicating this need for data collection is the ultrarare nature of inborn errors of metabolism that comprise the bulk of the NBS panel. Because most metabolic centers typically treat only a few patients with a given disorder, individual centers are unlikely to yield enough data to be meaningful, even if data are collected over many years. Ideally, data should be obtained from centers across a multitude of states that use different screening cutoffs and modalities, from a variety of practitioners who use varied follow-up and treatment methods, from large numbers of patients with a range of clinical presentations and genotypes, and from diverse patient populations. These data would then be used to determine optimal screening methods, to critically assess best clinical practices, and to help define the phenotypic spectrum among a heterogeneous patient population. The only way to achieve this is through large scale, multistate, multicenter collaborations, a notoriously intimidating prospect.

The Inborn Errors of Metabolism Information System (IBEM-IS), described in this issue of Genetics in Medicine, ${ }^{2}$ is a groundbreaking effort to gather and share information about the outcomes of infants with disorders detectable by NBS. It was initially developed to capture data from region 4 centers, but now it aptly includes multiple states, practitioners, and patients from diverse populations. In addition, there is an important collaboration between the Inborn Errors of Metabolism Collaborative and the Newborn Screening Translational Research Network to maximize the sharing of uniform data. The development of IBEM-IS faced enormous challenges, including how to use common data elements that are relatable to other databases and searches, how much and what types of data to capture, and what disorders to include in the database. The database itself posed novel issues relating to privacy, security, and data sharing across institutions and entities. At the site level, finding the personnel

${ }^{1}$ Department of Pediatrics, The Children's Hospital at Montefiore and the Albert Einstein College of Medicine, Bronx, New York, USA. Correspondence: Melissa P. Wasserstein (mwassers@montfiore.org)

Submitted 6 June 2016; accepted 7 June 2016; advance online publication 25 August 2016. doi:10.1038/gim.2016.99 
and time to obtain institutional review board approvals, receive consent from families, and enter data was an undoubtedly formidable prospect. Despite these hurdles, IBEM-IS has not only achieved but surpassed its goals, and it is clear that the NBS community recognizes its importance. At present, more than 30 centers across the United States are active participants. Data collection has started for most of the disorders on the Recommended Uniform Screening Panel. Almost 2,000 patients have consented to participate, and more than half a million data points have been entered. The remarkable team leading this project has proven that worthy ideas coupled with thoughtful planning and affable teamwork can make amazing things happen.

So far, the efforts have been fruitful. A report describing the use of the IBEM-IS to illustrate the outcomes of children with 3-methylcrotonyl-CoA carboxylase deficiency was recently published. ${ }^{3}$ The IBEM-IS database was also used to describe outcomes and genotype-phenotype correlations of individuals with very-long-chain acyl-CoA dehydrogenase deficiency. ${ }^{4}$ These are just the beginning; the potential of IBEM-IS to inform NBS practices is substantial. It will be exciting to see the diversity of clinical practices that are currently in use, to discover which are the most effective at improving outcomes, and to objectively determine whether early detection does in fact improve patient outcomes for particular disorders. In addition, the wide breadth of socioeconomic data in the IBEM-IS may help define the factors associated with patients being lost to follow-up, and may even help dictate paths to improve adherence.

Despite the positive interim results, concern remains about the long-term sustainability of the IBEM-IS. One challenge stems from the potential creation of similar but distinct databases to capture long-term outcome data about particular disorders. Because most centers will not have the capacity to participate in more than one database project, the already limited data will be split between competing projects, which will ultimately restrict the utility of such endeavors. After all, the power of the IBEM-IS lies in its numbers. Another challenge is maintaining the momentum to continue this important project. Not surprisingly, most metabolic treatment centers are challenged to find sufficient time and personnel to provide routine care; finding the bandwidth for patient recruitment and data entry, by necessity, takes lower priority. Maintaining the capacity to capture high-quality data requires the continued availability of funds to keep dedicated personnel focused on these projects. State legislative activity, funding sources, and advocacy efforts have largely focused on the "sexier" and more pressworthy issues of adding new disorders to NBS; it is as important for these stakeholders to focus this energy on keeping adequate funding available to ensure continued coordination and database maintenance of the IBEM-IS, and to allow sites to have dedicated personnel working on subject recruitment and data entry.

The goal of improving the outcome of infants with disorders screened by NBS using objective evidence to inform and guide care can be achieved only through large-scale data-collection activities such as IBEM-IS. This noble endeavor is important and timely, and it deserves long-term attention.

\section{DISCLOSURE}

The author declares no conflict of interest.

\section{REFERENCES}

1. Guthrie R, Susi A. A simple phenylalanine method for detecting phenylketonuria in large populations of newborn infants. Pediatrics 1963;32:338-343.

2. Berry SA, Leslie ND, Edick MJ, et al. Inborn Errors of Metabolism Collaborative: large-scale collection of data on long-term follow-up for newborn-screened conditions. Genet Med e-pub ahead of print 19 May, 2016.

3. Forsyth R, Vockley CW, Edick MJ, et al. Inborn errors of metabolism collaborative. Outcomes of cases with 3-methylcrotonyl-CoA carboxylase (3-MCC) deficiency -report from the Inborn Errors of Metabolism Information System. Mol Genet Metab 2016;118:15-20.

4. Pena LD, van Calcar SC, Hansen J, et al.; IBEMC. Outcomes and genotypephenotype correlations in 52 individuals with VLCAD deficiency diagnosed by NBS and enrolled in the IBEM-IS database. Mol Genet Metab; e-pub ahead of print 13 May 2016. 